\title{
Expression and prognostic impact of immune modulatory molecule PD-L1 in meningioma
}

\author{
Seunggu J. Han ${ }^{1}$, Gerald Reis ${ }^{2}$, Gary Kohanbash ${ }^{1}$, Shruti Shrivastav ${ }^{1}$, Stephen T. Magill' \\ Annette M. Molinaro ${ }^{1,3}$, Michael W. McDermott ${ }^{1}$, Philip V. Theodosopoulos ${ }^{1}$, Manish K. \\ Aghi $^{1}$, Mitchel S. Berger ${ }^{1}$, Nicholas A. Butowski ${ }^{1}$, Igor Barani ${ }^{4}$, Joanna J. Phillips ${ }^{1,2}$, Arie \\ Perry $^{1,2}$, and Hideho Okada ${ }^{1}$ \\ ${ }^{1}$ Department of Neurological Surgery, University of California, San Francisco, 505 Parnassus Ave, \\ M-779, San Francisco, CA 94143, USA \\ ${ }^{2}$ Department of Pathology, Division of Neuropathology, University of California, San Francisco, \\ San Francisco, CA, USA \\ ${ }^{3}$ Department of Epidemiology and Biostatistics, University of California, San Francisco, San \\ Francisco, CA, USA \\ ${ }^{4}$ Department of Radiation Oncology, University of California, San Francisco, San Francisco, CA, \\ USA
}

\section{Abstract}

While immunotherapy may offer promising new approaches for high grade meningiomas, little is currently known of the immune landscape in meningiomas. We sought to characterize the immune microenvironment and a potentially targetable antigen mesothelin across WHO grade I-III cases of meningiomas, and how infiltrating immune populations relate to patient outcomes.

Immunohistochemistry was performed on tissue microarrays constructed from 96 meningioma cases. The cohort included 16 WHO grade I, 62 WHO grade II, and 18 WHO grade III tumors. Immunohistochemistry was performed using antibodies against CD3, CD8, CD20, CD68, PD-L1, and mesothelin. Dual staining using anti-PD-L1 and anti-CD68 antibodies was performed, and automated cell detection and positive staining detection algorithms were utilized. Greater degree of PD-L1 expression was found in higher grade tumors. More specifically, higher grade tumors contained increased numbers of intratumoral CD68-, PD-L1+ cells $(\mathrm{p}=0.022)$, but did not contain higher numbers of infiltrating CD68+, PD-L1+ cells ( $\mathrm{p}=0.30$ ). Higher PD-L1+/CD68expression was independently predictive of worse overall survival in our cohort when accounting for grade, performance status, extent of resection, and recurrence history $(\mathrm{p}=0.014)$. Higher expression of PD-L1+/CD68- was also present in tumors that had undergone prior radiotherapy ( $\mathrm{p}$ $=0.024)$. Approximately quarter of meningiomas overexpressed mesothelin to levels equivalent to those found in pancreatic carcinomas and malignant mesotheliomas. The association with poor survival outcomes in our study suggests that PD-L1 may play a significant biologic role in the

Correspondence to: Seunggu J. Han.

Electronic supplementary material The online version of this article (doi:10.1007/s11060-016-2256-0) contains supplementary material, which is available to authorized users.

Conflict of interest The authors report no conflict of interests. 
aggressive phenotype of higher grade meningiomas. Thus, immunotherapeutic strategies such as checkpoint inhibition may have clinical utility in PD-L1 overexpressing meningiomas.

\section{Keywords}

Meningioma; PD-L1; B7-H1; Immunotherapy; Checkpoint; Lymphocyte; Macrophage

\section{Introduction}

Meningiomas represent the most common primary intra-cranial tumor found in adults, comprising roughly $36 \%$ of such tumors [1]. Although most meningiomas are classified by WHO as grade I, higher grade subsets exist, and grade II atypical meningiomas and grade III anaplastic/malignant meningiomas constitute $20-35 \%$ and approximately $3 \%$ of all meningiomas respectively [2]. Regardless of grade, the primary treatment modality for symptomatic and enlarging meningiomas currently is surgical resection. Higher WHO grade tumors exhibit aggressive clinical behavior, with grade II meningiomas having median progression free survival of approximately 7 years, and grade III tumors displaying frank malignant characteristics with overall survival of less than 3 years [2]. For these higher grade meningiomas, radiotherapy is commonly utilized as an adjuvant; however, robust prospective evidence supporting its use, and its exact role in the postoperative setting is currently lacking [3]. In addition, when these tumors recur after having failed surgery and radiotherapy, treatment options are severely limited and the clinical outcomes extremely poor [4]. Thus, there is a clear need for new effective therapies to serve as adjuvant or salvage agents for atypical and anaplastic meningiomas.

Refining our understanding of the complex interaction between the immune system and malignancies has led to the development of exciting novel immunotherapeutic approaches. Inhibitors of immune checkpoint pathways cytotoxic T lymphocyte antigen 4 (CTLA-4), and programmed death 1 (PD-1)/PD-ligand 1 (PD-L1) have shown promise for even advanced systemic malignancies such as metastatic melanoma, and recently was granted approval from the FDA [5-7]. While immunotherapy also offers promising potential avenues for meningiomas, little is currently known of the immune microenvironment in these tumors. Earlier studies have discovered evidence of macrophages and small populations of both $\mathrm{T}$ and B lymphocytes [8-11]; however, their significance and their relationship to clinical behavior have remained largely unclear. A recent report by Du et al. demonstrated that there were significantly fewer infiltrating $\mathrm{T}$ lymphocytes, including CD4+ and CD8+ T cells and cells expressing PD-1 within anaplastic meningiomas compared to lower grade tumors [12]. In addition, they found that PD-L1 is expressed in meningioma both at the protein and mRNA levels, and the levels of expression were higher in anaplastic cases [12]. Their analysis however failed to detect a significant relationship between PD-L1 levels and survival outcomes in their cohort consisting mostly of grade I meningiomas. We sought to further explore the prognostic significance of PD-L1 in meningiomas, investigate how PDL1 expression relates to the infiltrating immune cell populations, including CD68+ macrophages in a cohort enriched for grade II and III cases. 
Furthermore, we tested we tested for the presence of a potentially targetable antigen mesothelin in meningiomas. Mesothelin is a cell surface protein whose exact role remains unclear, but is likely involved in cell adhesion [13]. It is the target of focus of an early phase clinical trial in pancreatic carcinoma, ovarian carcinoma, and malignant mesothelioma utilizing chimeric antigen receptor (CAR) T-cells targeted against mesothelin (clinicaltrials.gov, NCT\#02159716). To explore whether mesothelin could serve as a potential targetable antigen in meningioma, mesothelin expression was also studied in our samples.

\section{Materials and methods}

Meningioma cases treated at the University of California, San Francisco Medical Center between 1989 and 2012 were selected based on the availability of sufficient tissue for immunohistochemical analysis as well as available clinical follow-up. In an attempt to optimize the translational potential for higher grade meningiomas, the cohort was enriched to capture all grade II and III encountered in the study period that had sufficient tissue. A random sample of grade I cases treated in the same time period was included for comparison in approximately equivalent numbers as grade III cases. Clinical information such as gender, tumor location, type of surgery, extent of resection, and treatment history was obtained from review of the medical records. Consultation cases were excluded. Recurrence was defined as the emergence of a new radiolographically defined lesion or lesions that required treatment. All pathologic diagnosis was confirmed by two of the authors (GR and AP) according to the criteria by the WHO. Approval for the study was obtained from the University of California San Francisco Committee for Human Research (Approval \#10-03204).

\section{Tissue microarray}

Tissue microarrays (TMA) were constructed using a semiautomatic tissue microarrayer (TMArryer, Pathology Devices, Westminster, MD). In brief, recipient blocks made using $5 \%$ agarose (A9539, Sigma Aldrich, St Louis, MO) in disposable base molds $(37 \times 24 \times 5$ mm; 62352-37, VWR, Radnor, PA). Agarose wafers were processed overnight in a Leica ASP tissue processor (Leica Biosystems, Buffalo Grove, IL) and subsequently embedded using conventional methods. Tissue from donor paraffin-embedded blocks of the select cases was sampled using a $1.5 \mathrm{~mm}$ punch (02110006; Pathology Devices, Westminster, MD). Duplicates were prepared, with each block containing 96 samples and including randomly selected control tissues. Recipient blocks were re-embedded and sectioned at 5 micrometer thickness for immunohistochemistry.

For mesothelin staining experiments, normal tissue TMA sections were obtained from Folio (cat\# ARY0HH0213, Powell, OH), normal peritoneum and pleura TMAs from Pantomics (cat\# GIP541, LUP541, Richmond, CA), multiple tumor TMA from Pantomics (cat\# MTU391, Richmond, CA) malignant mesothelioma from MyBioSource (cat\# MBS640483, San Diego, CA). 


\section{Immunohistochemistry}

The following primary antibodies were used in this study: CD3 (1:25, Clone: F7.2.38, Dako, Carpinteria, CA), CD8 (1:50, Clone C8/144B, Dako, Carpinteria, CA), CD20 (1:20, ab9475, Abcam, Cambridge, MA) CD68 (1:50, ab49777, Abcam, Cambridge, MA), PD-L1 (1:200, cat \#13684, Cell Signaling Technology, Danvers, MA), mesothelin (1:50, sc-271540, Santa Cruz Biotechnology, Dallas TX).

Slides were deparaffinized in xylene and rehydrated in serial baths of graded ethanol (100\%, $95 \%)$. Heat induced epitope retrieval was performed with a citrate buffer (Target Retrieval Solution Citrate pH6, Dako, Carpinteria, CA) in a decloaking chamber (Biocare Medical, Concord, CA) at $20 \mathrm{psi}$ for $10 \mathrm{~min}$, followed by $30 \mathrm{~min}$ of cooling at room temperature. Endogenous peroxidase activity was quenched in $3 \%$ hydrogen peroxide for $10 \mathrm{~min}$ at room temperature. Blocking of non-specific binding of primary antibody was performed using a solution of $5 \%$ normal goat serum (Cell Signaling Technology, Danvers, MA). Slides were incubated with primary antibodies at $4^{\circ}$ overnight. The sections were then incubated at room temperature for $1 \mathrm{~h}$ with secondary horseradish peroxidase conjugated goat antibodies (Signal-Stain Boost IHC Detection Reagent HRP Mouse and HRP Rabbit, Cell Signaling Technology, Danvers, MA). Visualization was performed with chromagen diaminobenzidine (DAB) (SignalStain DAB Substrate Kit, Cell Signaling Technology, Danvers, MA). Counterstaining was performed with hematoxylin (Hematoxylin QS, Vector Laboratories, Burlingame, CA), and the slides were mounted with a cover slip (Cytoseal 60, Thermo Scientific, Waltham, MA).

For dual target staining, following primary antibody incubation, the slides were incubated with secondary goat anti-mouse and goat anti-rabbit antibodies, conjugated to the fluorochromes Alexa Fluor 488 and Alexa Fluor 555, respectively, at 1:200 dilution for $1 \mathrm{~h}$ at room temperature (Lot 1557766 and Lot 1600203, Life Technologies, Carlsbad, CA). The slides were cover-slipped with a mountant containing DAPI (ProLong Gold antifade reagent with DAPI, Life Technologies, Carlsbad, CA). All staining was completed in duplicate.

\section{Image acquisition}

Stained sections were imaged with Zeiss Axio Imager 2 microscope (Zeiss, Oberkochen, Germany). Image acquisition was completed while using identical camera settings of exposure time and white balance throughout all sections stained with the same antibody, with the aid of an automated scanning software, TissueFAXS (TissueGnostics, Vienna, Austria).

\section{Image analysis}

Quantification of positive staining was performed with the image analysis software StrataQuest (TissueGnostics, Vienna, Austria). In brief, for sections stained for each target epitope, a set of analysis algorithms were generated by a trained user. First, a cell detection algorithm identified nucleated cells based on hematoxylin or DAPI staining, which incorporated nuclear size, shape, and intensity of the signal. The next layer of analysis was designed to detect the intensity of staining in the DAB color or the FITC/Texas Red channels associated with each detected cell. Tabulation of positive cells in each TMA was determined 
by a threshold set by the user based on the detection of signal intensity. Once each algorithm was generated and tested to appropriately identify positive cells, it was applied to all TMA images to calculate a total number of positive cells per area, as well as percentage of positively staining cells in the entire sample.

\section{Statistical analysis}

Expression of different markers between groups was compared using the $t$-test or ANOVA. Survivals were estimated using the Kaplan-Meier method. Comparisons of survivals used the Log-rank test. Mutivariate cox proportional hazards were used for modeling risks of progression or death based on prognostic variables. Splits for continuous predictive variables were generated using classification and regression trees. Statistical analyses were performed using JMP software (SAS, Cary, Canada).

\section{Results}

Patients

The cohort included 96 total patients with16 grade I, 62 grade II, and 18 grade III tumors. The median age was 60 at diagnosis, and 57 patients (59\%) were female. Twenty-five cases (26\%) were recurrent cases, with 23 cases $(24 \%)$ having received prior radiation therapy as adjuvant treatment for their meningioma. Table 1 summarizes the patients' baseline characteristics and prior therapies.

\section{Infiltrating immune cell populations}

Our immunohistochemistry analysis confirmed the presence of $\mathrm{T}$ lymphocytes in majority of cases. We found a median of $114 \mathrm{CD} 3+$ lymphocytes per core $\left(64 / \mathrm{mm}^{2}\right)$, and median of 126 CD8 + lymphocytes per core $\left(71 / \mathrm{mm}^{2}\right)$ across all grades of tumors (Table 2). B lymphocytes were also present, but in much smaller numbers, with median of $3.5 \mathrm{CD} 20+$ cells per core $\left(2 / \mathrm{mm}^{2}\right)$. We did not note a difference in numbers of infiltrating any subpopulation of lymphocytes based on the grade of tumor, or on prior treatment history. CD68+ infiltrating macrophages were more abundant, and there was median of 3752 CD68+ cells per core $\left(2120 / \mathrm{mm}^{2}\right)$.

\section{Expression of PD-L1 in meningioma}

PD-L1 expression was calculated as a percentage of positively staining cells across the core. Across all grades, the mean percent of PD-L1+ cells was 3.51\% (Range 0-85.4\%, Table 2). Higher expression of PD-L1 was found in higher grade tumors. On average, PD-L1 expression was found in $0.91 \%$ of cells for grade I, $2.63 \%$ of cells for grade II, and $8.84 \%$ of cells for grade III meningiomas (ANOVA, $\mathrm{p}=0.039$, Fig. 1). Previous treatment with prior radiotherapy was also correlated with high PD-L1 expression; tumors that had undergoing prior radiotherapy contained an average of $6.79 \%$ PD-L1+ cells, while tumors that did not had $2.50 \%$ (Student's $t$-test, $\mathrm{p}=0.041$, Fig. 1). PD-L1 expression did not appear to have significant direct or inverse relationship to the degree of infiltrating CD3+, CD8+, CD20+ or CD68+ cells. 


\section{PD-L1 expression in infiltrating macrophages}

To clarify the source of PD-L1 expression in meningioma and address the possibility of infiltrating macrophage populations being a significant source of PD-L1 within the tumor, we performed costaining experiments for PD-L1 and CD68 (Fig. 2). Although the majority of PD-L1+ cells were also negative for CD68 across all cores, suggesting that PD-L1 is likely expressed by the tumor cells themselves, in a subset of tumors, most of the PD-L1+ cells were also CD68+. In 36 samples (43.3\%), the CD68+ infiltrating macrophage population was the main source of PD-L1 expression within the tumor tissue, i.e. more than $50 \%$ of the total PD-L1+ cells was also CD68+.

\section{PD-L1 expression is predictive of poor overall survival}

The median overall survival of the entire cohort was 11.8 years. A cox proportional hazards model was performed accounting for potential prognostic variables such as age, performance status, extent of resection, grade, recurrent status, along with numbers of lymphocytic/ macrophage infiltrates and level of PD-L1+ cells. On univariate analysis, Karnofsky Performance Scale (KPS), grade, and proportions of PD-L1+ cells and PD-L1+/CD68- cells were significant predictors of overall survival, while percent of PD-L1+/CD68+ cells was not. In addition, presence of PD-L1+/CD68- cells was more predictive of worse survival than total PD-L1 + levels (HR per $1 \%$ expressing cells change: 1.037 vs. 1.015, respectively), suggesting that the prognostic impact of PD-L1 seem primarily driven by PDL1 expression on CD68- meningioma cells. When accounting for age, KPS, grade and extent of resection in a multivariate model, PD-L1+/CD68- expression remained significantly predictive of worse overall survival (HR 1.263 per $1 \%$ of cells, $p=0.014$, Table 3). When the same multivariate model was built using all PD-L1 + cells, total PD-L1 + population was no longer predictive of overall survival $(\mathrm{p}=0.179)$.

Classification and regression trees analysis revealed that presence of $>0.12 \%$ of PD-L1+l CD68- cells as the most significant cut-off predicting worse overall survival (log rank, $\mathrm{p}=$ 0.027 , Figure S1).

\section{CD20+ cell infiltration predicts favorable progression free survival}

Median progression free survival was 6.4 years. Cox proportional hazards modeling utilizing same prognostic variable as above revealed grade and CD20+ infiltrates as significant predictors of progression free survival in univariate modeling. Multivariate analysis accounting for grade and extent of resection revealed infiltration with CD20+ cells was independently predictive of improved progression free survival (HR 0.966 per CD20+ cell in core, $\mathrm{p}=0.041$, Table 3).

\section{Mesothelin as a potential targetable antigen}

The median percent of cells staining positively for mesothelin across all cores was $3.93 \%$; however, ten percent of cores contained $>35.7 \%$ of mesothelin positive cells (Figure S2). Using same staining conditions, sections of normal organs and multiple types of solid organ malignancies were also stained for mesothelin. Among normal organs, the percent of cells staining positive for mesothelin ranged from 0.87 to $31.8 \%$. Organs with $>10 \%$ staining included pleura (31.8\%), lymph node $(21.6 \%)$, stomach $(16.2 \%)$ and lung $(15.4 \%)$. 
Positive staining was found $0.2-49.3 \%$ of solid organ tumors. Tumors with the most mesothelin expression were ovarian adenocarcinoma (49.3\%), colon adenocarcinoma (19.9\%), stomach adenocarcinoma (14.7\%), pancreatic carcinoma (13.8\%), and mesothelioma (12.2\%). In our meningioma samples, the 75th percentile of mesothelin positive staining was $17 \%$, indicating that a quarter of the tumors expressed mesothelin levels near those found in pancreatic carcinoma and mesothelioma. Supplementary Table 1 summarizes the results of mesothelin staining.

\section{Discussion}

Our results demonstrate presence of infiltrating immune cells in meningioma, with CD68+ cells in relative abundance, fewer CD3+ and CD8+ lymphocytes, and rare CD20+ lymphocytes. Although the relative distribution of tumor infiltrating immune cells is consistent with those seen in other tumors of the central nervous system, such as glioblastoma, primary CNS lymphoma, and brain metastases [14-17], it is difficult to say how the degree of immune cell infiltration in meningiomas compare to those in these other tumors, as the method of detection varied. Traditional method of quantifying tumor infiltrating lymphocytes involves a semi-quantitative four-tiered system based on manual blinded assessment of low and high powered fields [18]. Our analysis minimizes biases by sampling the entire tissue section, and provides an objective fully quantitative count of the immune cell population using a software algorithm. Thus, the results obtained in our analysis is a count of cells across an area of tissue, rather than a category of "sparse" to "very dense" infiltration.

We also confirmed previous findings that PD-L1 is present in meningiomas, and more expression is found in higher grade tumors. In addition, PD-L1 expression, particularly among cells that were negative for CD68, was predictive of poor overall survival in our cohort. Prior investigation of PD-L1 expression in meningioma by Du et al. did not detect any prognostic impact of PD-L1 expression [12]. One main difference in our analysis was the inclusion of double staining of PD-L1 with CD68, and this analysis revealed that only the PD-L1 expression on CD68- cells was an independent predictor of survival outcome. On the other hand, total PD-L1 expression itself was not an independent prognostic indicator of outcome, which was consistent to findings of Du et al. [12].

This finding may also be explained in part by the types of cases included for analyses. The cohort evaluated by Du et al. included consecutive meningioma cases of all grades at a single institution, and hence $67 \%$ of their TMA consisted of grade I tumors [12]. If the degree of PD-L1 expression is predictive of poor survival only among grade II/III tumors, this effect may not be detectable in a series consisting of mostly grade I tumors, as the prognostic impact maybe diluted. In addition, in a cohort of mostly grade I tumors, it may be likely that there were many censored samples in the survival analysis. If this is the case, it may explain why our analysis was able to detect a prognostic impact of PD-L1 expression, as the cases represented in our TMA were enriched for grade II/III tumors.

PD-1/PD-L1 axis-driven immunosuppression has been implicated in multiple tumor types, including tumors of the central nervous system such as glioblastoma, chordoma, and primary 
central nervous system lymphoma [17, 19-21]. In many of these cases, PD-L1 expression was also observed on tumor infiltrating macrophages, including in malignant gliomas. In malignant gliomas, PD-L1 expressing tumor infiltrating macrophages are associated with worse outcomes, and are able induce an immunosuppressive phenotype by inducing $\mathrm{T}$ cell apoptosis [22, 23]. Our study discovered PD-L1 expressing CD68+ tumor infiltrating cells in a significant subset of meningiomas cases. However, the biologic impact of these CD68+/PD-L1+ cells seems unclear as their presence was not correlated with pathologic grade nor was it prognostic of overall or progression free survival in our cohort. On the other hand, we cannot be certain that the CD68-/PD-L1+ population seen within tumor tissue solely represents tumor cells. Dual staining with meningioma specific markers including vimentin and EMA were attempted, however, appropriate antibody combinations and staining conditions could not be achieved to allow for confident reading of PD-L1 expression signal.

In our series, PD-L1 expression was also significantly higher in recurrent tumors and after prior radiation therapy. This association was likely driven by the increase in PD-L1+/CD68cells, as the degree of PD-L1+/CD68+ cells did not significantly differ based on prior radiotherapy. This finding was not surprising, as radiotherapy has been shown to upregulate PD-L1 in other tumor types, likely through release of interferon-gamma [24]. In a recent report, Moliterno et al., based on a retrospective series, observed that de novo grade III tumors seem to have more favorable survivals compared to those that progressed from a lower grade tumor, independent of extent of resection [25]. In our cohort, although prior treatment status correlated with higher PD-L1 expression, PD-L1 expression on CD68- cells was significantly predictive of poor overall survival while recurrent status alone was not. It would be of interest to see if the de novo cases in Moliterno et al.'s series indeed contained lower levels of PD-L1. Nonetheless, the association with decreased overall survival suggests that PD-L1 may play a biologically significant role in the aggressive phenotype of meningiomas that recur after radiotherapy. In addition, it may be worth exploring whether PD-1/PD-L1 axis blockade would have clinical utility in radiation failed tumors, and whether combination therapy with radiotherapy would be able to abrogate the potential impacts of this PD-L1 upregulation.

Given that CD20 + cells were detected with relative infrequency in the vast majority of samples studied, its association with progression free survival was somewhat a surprising finding. Prior studies have also described the rare presence CD20+ B cells within meningiomas, and consistent with Du et al.'s experience, we also did not detect a relationship between $\mathrm{CD} 20+$ infiltrates and tumor grade [12]. In addition, the ultimate biologic impact of infiltrating CD20+ cells is made less clear by our finding that presence CD20+ cells was not predictive of improved overall survival. Still, antibody mediated immunity may have a larger role in the immune-tumor interaction in meningiomas, given the lack of a blood brain barrier around these tumors, compared to other locations in the central nervous system. In a canine model of meningioma, Andersen et al. successfully induced an antibody mediated response to an autologous tumor cell lysate vaccine [26]. In their analysis, infiltration of plasma cells were also seen post vaccination, and isolated tumorreactive antibodies were capable of cell-mediated induction of antibody-dependent killing of autologous tumor cells. Further characterization of the infiltrating B cell population, 
including their activation status, is clearly needed to gain further insights into the impact of the humoral immune system's role in meningioma progression and proliferation.

Mesothelin was explored as a potential targetable antigen in meningioma, as an early phase clinical trial is currently exploring the use of CAR T cells targeted against mesothelin for pancreatic carcinoma, ovarian carcinoma, and malignant mesothelioma (clinicaltrials.gov, NCT\#02159716). Mesothelin is also expressed in many normal organs, with higher levels in connective tissue such as pleura and the gastrointestinal system. The exact role of mesothelin remains unclear, as knockout mice develop and reproduce normally [27]. Based on our experience, tumors originating from solid organs generally seem to upregulate mesothelin expression relative to normal levels found in that particular organ. Mesothelin has been shown to be also expressed to varying degrees in meningiomas of all grades, while very few normal meninges expressed mesothelin [13]. Our analysis confirms these previous findings, and overall levels of expression appears similar to the previously published reports. Most tumors showed little to no staining, but a small subset had significant levels of expression, in up to $99 \%$ of cells. Approximately a quarter of the tumors expressed mesothelin levels near those found in pancreatic carcinoma and mesothelioma ( 15\%). Thus, in a subset of meningiomas with high mesothelin expression, a similar strategy of targeting mesothelin could be potentially utilized.

Our analysis used an automated quantification of all stained sections. The StrataQuest imaging analysis software, once trained, allowed for objective quantification of positive staining. In addition, the software applied the same algorithm of cell detection and positive staining quantification to all cores on the TMA. We feel that this method provided a rapid, reproducible and unbiased method of quantification of large number of samples when compared to manual counting or scoring.

Limitations of our analysis include those inherent to TMA based studies, such as the possibility that the cores may miss the intratumoral heterogeneity with regards to the immune microenvironment. Selection bias may have also been introduced by enriching our cohort for grade II/III tumors and cases that had sufficient tissue to generate cores.

\section{Conclusion}

In our cohort PD-L1 expression, specifically by CD68- cells, and not in CD68+ infiltrating cells, was associated with higher grade tumors and prior treatment with radiotherapy. The presence of PD-L1+/CD68- cells was independently prognostic of poor overall survival in our study, suggesting PD-L1 in this population may play a significant biologic role in the aggressiveness of higher grade and radiotherapy failed meningiomas. Thus, immunotherapeutic approaches such as checkpoint inhibitors of PD-1/PD-L1 axis, or targeting mesothelin through vaccines or engineered $\mathrm{T}$ cells may have clinical utility for aggressive high grade tumors.

\section{Supplementary Material}

Refer to Web version on PubMed Central for supplementary material. 


\section{Acknowledgments}

Funding Dr. Han is supported by the Neurosurgery Research and Education Foundation Research Fellowship and the Hanson Foundation for Meningioma Research.

\section{References}

1. Ostrom QT, Gittleman H, Liao P, Rouse C, Chen Y, Dowling J, Wolinsky Y, Kruchko C, BarnholtzSloan J. CBTRUS statistical report: primary brain and central nervous system tumors diagnosed in the United States in 2007-2011. Neuro-oncology. 2014; 16(Suppl 4):iv1-i63. DOI: 10.1093/neuonc/ nou223 [PubMed: 25304271]

2. Rogers L, Barani I, Chamberlain M, Kaley TJ, McDermott M, Raizer J, Schiff D, Weber DC, Wen PY, Vogelbaum MA. Meningiomas: knowledge base, treatment outcomes, and uncertainties. A RANO review. J Neurosurg. 2015; 122(1):4-23. DOI: 10.3171/2014.7.JNS131644 [PubMed: 25343186]

3. Jenkinson MD, Weber DC, Haylock BJ, Mallucci CL, Zakaria R, Javadpour M. Atypical meningoma: current management dilemmas and prospective clinical trials. J Neurooncol. 2015; 121(1):1-7. DOI: 10.1007/s11060-014-1620-1 [PubMed: 25258253]

4. Kaley T, Barani I, Chamberlain M, McDermott M, Panageas K, Raizer J, Rogers L, Schiff D, Vogelbaum M, Weber D, Wen P. Historical benchmarks for medical therapy trials in surgery- and radiation-refractory meningioma: a RANO review. Neuro-oncology. 2014; 16(6):829-840. DOI: 10.1093/neuonc/not330 [PubMed: 24500419]

5. Robert C, Long GV, Brady B, Dutriaux C, Maio M, Mortier L, Hassel JC, Rutkowski P, McNeil C, Kalinka-Warzocha E, Savage KJ, Hernberg MM, Lebbe C, Charles J, Mihalcioiu C, Chiarion-Sileni V, Mauch C, Cognetti F, Arance A, Schmidt H, Schadendorf D, Gogas H, Lundgren-Eriksson L, Horak C, Sharkey B, Waxman IM, Atkinson V, Ascierto PA. Nivolumab in previously untreated melanoma without BRAF mutation. N Engl J Med. 2015; 372(4):320-330. DOI: 10.1056/ Nejmoa1412082 [PubMed: 25399552]

6. Hodi FS, O’Day SJ, McDermott DF, Weber RW, Sosman JA, Haanen JB, Gonzalez R, Robert C, Schadendorf D, Hassel JC, Akerley W, van den Eertwegh AJ, Lutzky J, Lorigan P, Vaubel JM, Linette GP, Hogg D, Ottensmeier CH, Lebbe C, Peschel C, Quirt I, Clark JI, Wolchok JD, Weber JS, Tian J, Yellin MJ, Nichol GM, Hoos A, Urba WJ. Improved survival with ipilimumab in patients with metastatic melanoma. N Engl J Med. 2010; 363(8):711-723. DOI: 10.1056/NEJMoa1003466 [PubMed: 20525992]

7. Robert C, Ribas A, Wolchok JD, Hodi FS, Hamid O, Kefford R, Weber JS, Joshua AM, Hwu WJ, Gangadhar TC, Patnaik A, Dronca R, Zarour H, Joseph RW, Boasberg P, Chmielowski B, Mateus C, Postow MA, Gergich K, Elassaiss-Schaap J, Li XN, Iannone R, Ebbinghaus SW, Kang SP, Daud A. Anti-programmed-death-receptor-1 treatment with pembrolizumab in ipilimumab-refractory advanced melanoma: a randomised dose-comparison cohort of a phase 1 trial. The Lancet. 2014; 384(9948):1109-1117. DOI: 10.1016/S0140-6736(14)60958-2

8. Domingues PH, Teodosio C, Ortiz J, Sousa P, Otero A, Maillo A, Barcena P, Garcia-Macias MC, Lopes MC, de Oliveira C, Orfao A, Tabernero MD. Immunophenotypic identification and characterization of tumor cells and infiltrating cell populations in meningiomas. Am J Pathol. 2012; 181(5):1749-1761. DOI: 10.1016/j.ajpath.2012.07.033 [PubMed: 22982440]

9. Rossi ML, Cruz Sanchez F, Hughes JT, Esiri MM, Coakham HB. Immunocytochemical study of the cellular immune response in meningiomas. J Clin Pathol. 1988; 41(3):314-319. [PubMed: 3258871]

10. Fang L, Lowther DE, Meizlish ML, Anderson RC, Bruce JN, Devine L, Huttner AJ, Kleinstein SH, Lee JY, Stern JN, Yaari G, Lovato L, Cronk KM, O'Connor KC. The immune cell infiltrate populating meningiomas is composed of mature, antigen-experienced $\mathrm{T}$ and B cells. Neurooncology. 2013; 15(11):1479-1490. DOI: 10.1093/neuonc/not110 [PubMed: 23978377]

11. Baia GS, Caballero OL, Ho JS, Zhao Q, Cohen T, Binder ZA, Salmasi V, Gallia GL, QuinonesHinojosa A, Olivi A, Brem H, Burger P, Strausberg RL, Simpson AJ, Eberhart CG, Riggins GJ. NY-ESO-1 expression in meningioma suggests a rationale for new immunotherapeutic approaches. Cancer Immunol Res. 2013; 1(5):296-302. DOI: 10.1158/2326-6066.CIR-13-0029 [PubMed: 24777967] 
12. Du Z, Abedalthagafi M, Aizer AA, McHenry AR, Sun HH, Bray MA, Viramontes O, Machaidze R, Brastianos PK, Reardon DA, Dunn IF, Freeman GJ, Ligon KL, Carpenter AE, Alexander BM, Agar NY, Rodig SJ, Bradshaw EM, Santagata S. Increased expression of the immune modulatory molecule PD-L1 (CD274) in anaplastic meningioma. Oncotarget. 2015; 6(7):4704-4716. [PubMed: 25609200]

13. Johnson MD, Vito F, O'Connell MJ. Mesothelin expression in the leptomeninges and meningiomas. J Histochem Cytochem. 2008; 56(6):579-585. DOI: 10.1369/jhc.2008.950477 [PubMed: 18347077]

14. Berghoff AS, Fuchs E, Ricken G, Mlecnik B, Bindea G, Spanberger T, Hackl M, Widhalm G, Dieckmann K, Prayer D, Bilocq A, Heinzl H, Zielinski C, Bartsch R, Birner P, Galon J, Preusser M. Density of tumor-infiltrating lymphocytes correlates with extent of brain edema and overall survival time in patients with brain metastases. Oncoimmunology. 2016; 5(1):e1057388.doi: 10.1080/2162402X.2015.1057388 [PubMed: 26942067]

15. Berghoff AS, Kiesel B, Widhalm G, Rajky O, Ricken G, Wohrer A, Dieckmann K, Filipits M, Brandstetter A, Weller M, Kurscheid S, Hegi ME, Zielinski CC, Marosi C, Hainfellner JA, Preusser M, Wick W. Programmed death ligand 1 expression and tumor-infiltrating lymphocytes in glioblastoma. Neuro-oncology. 2015; 17(8):1064-1075. DOI: 10.1093/neuonc/nou307 [PubMed: 25355681]

16. Berghoff AS, Ricken G, Widhalm G, Rajky O, Dieckmann K, Birner P, Bartsch R, Holler C, Preusser M. Tumour-infiltrating lymphocytes and expression of programmed death ligand 1 (PDL1) in melanoma brain metastases. Histopathology. 2015; 66(2):289-299. DOI: 10.1111/his.12537 [PubMed: 25314639]

17. Berghoff AS, Ricken G, Widhalm G, Rajky O, Hainfellner JA, Birner P, Raderer M, Preusser M. PD1 (CD279) and PD-L1 (CD274, B7H1) expression in primary central nervous system lymphomas (PCNSL). Clin Neuropathol. 2014; 33(1):42-49. [PubMed: 24359606]

18. Dahlin AM, Henriksson ML, Van Guelpen B, Stenling R, Oberg A, Rutegard J, Palmqvist R. Colorectal cancer prognosis depends on T-cell infiltration and molecular characteristics of the tumor. Mod Pathol. 2011; 24(5):671-682. DOI: 10.1038/modpathol.2010.234 [PubMed: 21240258]

19. Han SJ, Ahn BJ, Waldron JS, Yang I, Fang S, Crane CA, Pieper RO, Parsa AT. Gamma interferonmediated superinduction of B7-H1 in PTEN-deficient glioblastoma: a paradoxical mechanism of immune evasion. Neuroreport. 2009; 20(18):1597-1602. DOI: 10.1097/WNR.0b013e32833188f7 [PubMed: 19875977]

20. Parsa AT, Waldron JS, Panner A, Crane CA, Parney IF, Barry JJ, Cachola KE, Murray JC, Tihan T, Jensen MC, Mischel PS, Stokoe D, Pieper RO. Loss of tumor suppressor PTEN function increases B7-H1 expression and immunoresistance in glioma. Nat Med. 2007; 13(1):84-88. DOI: 10.1038/ nm1517 [PubMed: 17159987]

21. Mathios D, Ruzevick J, Jackson CM, Xu H, Shah S, Taube JM, Burger PC, McCarthy EF, Quinones-Hinojosa A, Pardoll DM, Lim M. PD-1, PD-L1, PD-L2 expression in the chordoma microenvironment. J Neurooncol. 2015; 121(2):251-259. DOI: 10.1007/s11060-014-1637-5 [PubMed: 25349132]

22. Bloch O, Crane CA, Kaur R, Safaee M, Rutkowski MJ, Parsa AT. Gliomas promote immunosuppression through induction of B7-H1 expression in tumor-associated macrophages. Clin Cancer Res. 2013; 19(12):3165-3175. DOI: 10.1158/1078-0432.CCR-12-3314 [PubMed: 23613317]

23. Rodrigues JC, Gonzalez GC, Zhang L, Ibrahim G, Kelly JJ, Gustafson MP, Lin Y, Dietz AB, Forsyth PA, Yong VW, Parney IF. Normal human monocytes exposed to glioma cells acquire myeloid-derived suppressor cell-like properties. Neuro-oncology. 2010; 12(4):351-365. DOI: 10.1093/neuonc/nop023 [PubMed: 20308313]

24. Bernstein MB, Garnett CT, Zhang H, Velcich A, Wattenberg MM, Gameiro SR, Kalnicki S, Hodge JW, Guha C. Radiation-induced modulation of costimulatory and coinhibitory T-cell signaling molecules on human prostate carcinoma cells promotes productive antitumor immune interactions. Cancer Biother Radiopharm. 2014; 29(4):153-161. DOI: 10.1089/cbr.2013.1578 [PubMed: 24693958] 
25. Moliterno J, Cope WP, Vartanian ED, Reiner AS, Kellen R, Ogilvie SQ, Huse JT, Gutin PH. Survival in patients treated for anaplastic meningioma. J Neurosurg. 2015; doi: 10.3171/2014.10.JNS14502

26. Andersen BM, Pluhar GE, Seiler CE, Goulart MR, SantaCruz KS, Schutten MM, Meints JP, O'Sullivan MG, Bentley RT, Packer RA, Thomovsky SA, Chen AV, Faissler D, Chen W, Hunt MA, Olin MR, Ohlfest JR. Vaccination for invasive canine meningioma induces in situ production of antibodies capable of antibody-dependent cell-mediated cytotoxicity. Cancer Res. 2013; 73(10): 2987-2997. DOI: 10.1158/0008-5472.CAN-12-3366 [PubMed: 23471847]

27. Bera TK, Pastan I. Mesothelin is not required for normal mouse development or reproduction. Mol Cell Biol. 2000; 20(8):2902-2906. [PubMed: 10733593] 

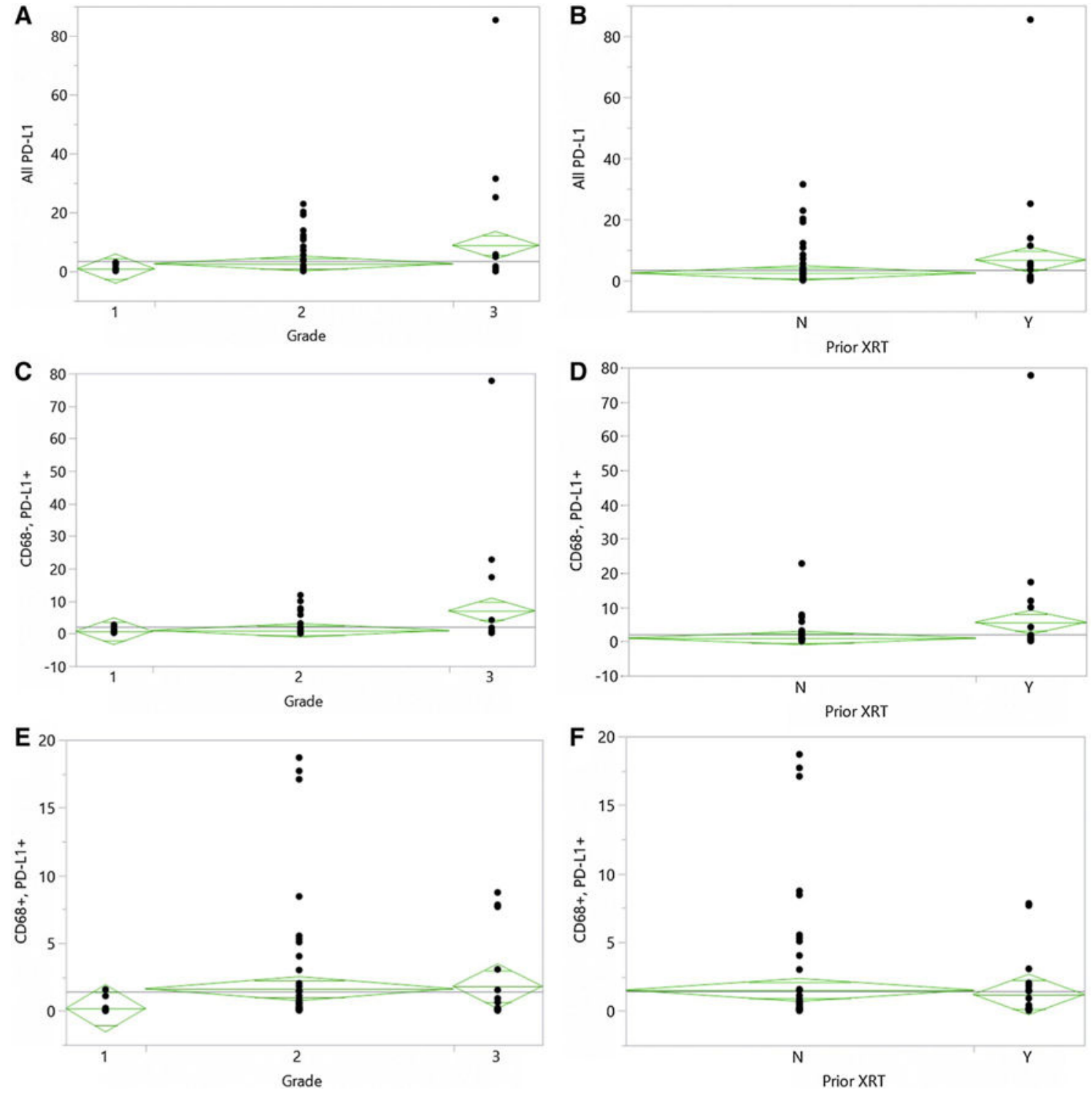

Fig. 1.

PD-L1 Expression based on tumor grade and prior radiotherapy. On average, PD-L1 expression was found in $0.91 \%$ of cells for grade I, $2.63 \%$ of cells for grade II, and $8.84 \%$ of cells for grade III meningiomas (ANOVA, $\mathrm{p}=0.039$, a). Tumors that had undergone prior radiotherapy contained an average of $6.79 \%$ PD-L1+ cells, while tumors that did not had $2.50 \%$ (Student's $t$-test, $\mathrm{p}=0.041, \mathbf{b}$ ). There were significantly greater numbers of PD-L1+/ CD68- cells in higher grade tumors (ANOVA, $\mathrm{p}=0.022$, c), and tumors that had undergone prior radiotherapy $(t$-test, $\mathrm{p}=0.024, \mathbf{d})$. There was no significant difference in numbers of infiltrating PD-L1+/CD68+ cells between tumors of different grades (ANOVA, $p=0.30$, e), and based on prior radiotherapy $(t$-test, $\mathrm{p}=0.71, \mathbf{f})$. Boxes represent mean and standard error 
A

B

Fig. 2.

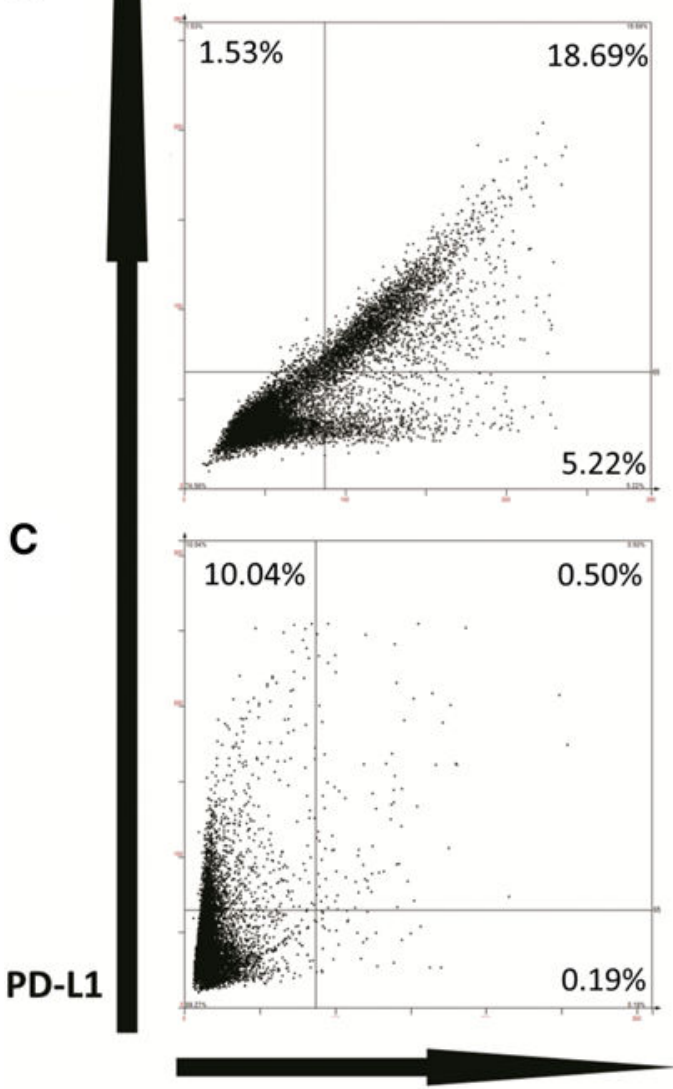

CD68
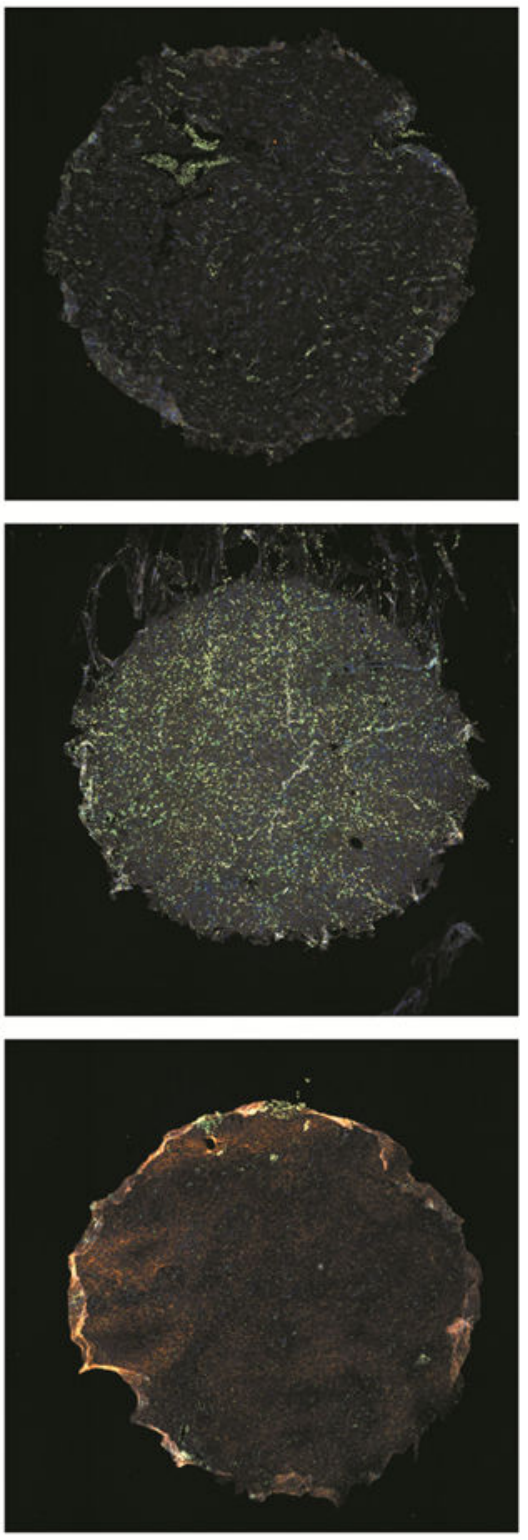

Patterns of PD-L1 Expression. Immunohistochemistry of double staining for PD-L1 and CD68 in representative WHO grade I (a), grade II (b), and grade III (c) tumors. Left panels show scatterplot generated by StratQuest image analysis software, based on mean staining intensity in FITC (CD68, x-axis) and Texas Red (PD-L1, y-axis) channels within each identified cell. Each dot represents one cell. Dividing lines within the plot represent intensity threshold used to consider positive signal in each channel. Although the scales of the axes appear different across the three representative samples, same staining intensity thresholds were used for all cores to determine positivity in each channel. Right panels show corresponding stained sections: DAPI (nuclear stain) in blue, FITC (CD68) in green, and 
Texas Red (PD-L1) in orange. Sample a shows representative tumor with very low overall PD-L1, with minority of cells expressing CD68. Sample b shows a significant PD-L1 + population that is also CD68+, as well as a separate population of CD68+/PD-L1- cells. Sample c demonstrates PD-L1 expression that is predominantly within CD68- cells. Core diameter is $1.5 \mathrm{~mm}$ 


\section{Table 1}

Clinical characteristics of patients

\begin{tabular}{|c|c|c|}
\hline \multirow[t]{2}{*}{ Characteristic } & \multicolumn{2}{|c|}{$\underline{\text { Entire population }(n=96}$} \\
\hline & $\mathbf{n}$ & $\%$ \\
\hline Age at diagnosis, median (range) & $59.8(18.7-86.5)$ & \\
\hline \multicolumn{3}{|l|}{ Gender } \\
\hline $\mathrm{F}$ & 57 & 59.4 \\
\hline M & 39 & 40.6 \\
\hline \multicolumn{3}{|l|}{ Karnofsky performance status } \\
\hline 90 & 3 & 3.12 \\
\hline 80 & 19 & 19.8 \\
\hline 70 & 23 & 24.0 \\
\hline$<70$ & 19 & 19.8 \\
\hline Unknown & 32 & \\
\hline \multicolumn{3}{|l|}{ Grade } \\
\hline I & 16 & 16.7 \\
\hline II & 62 & 64.6 \\
\hline III & 18 & 18.8 \\
\hline \multicolumn{3}{|l|}{ Recurrence status } \\
\hline Recurrent tumor & 25 & 26.0 \\
\hline Newly diagnosed tumor & 71 & 74.0 \\
\hline \multicolumn{3}{|l|}{ Prior radiotherapy } \\
\hline Yes & 23 & 24.0 \\
\hline No & 73 & 76.0 \\
\hline \multicolumn{3}{|l|}{ Extent of resection } \\
\hline Gross total resection & 36 & 43.3 \\
\hline Subtotal resection & 47 & 56.6 \\
\hline
\end{tabular}

Baseline clinical characteristics of patients whose tumors were included in the TMA 


\section{Table 2}

Degree of infiltrating immune cell populations

\begin{tabular}{lllll}
\hline & Mean (range) & 25th percentile & Median & 75th percentile \\
\hline & Cells per core & & & \\
CD3+ & $194(0-1512)$ & 50 & 114.5 & 254 \\
CD8+ & $188(0-956)$ & 52.75 & 126.5 & 244.75 \\
CD20+ & $11.0(0-220)$ & 1 & 3.5 & 8 \\
CD68+ & $4204(596-13,781)$ & 2337.25 & 3752 & 5163 \\
& Percent of cells staining positive & & & \\
PD-L1+ & $3.5(0-85.4)$ & 0.063 & 0.41 & 1.9 \\
PD-L1+/CD68+ & $1.43(0-18.7)$ & 0 & 0.90 & 5.14 \\
PD-L1+/CD68- & $2.08(0-77.69)$ & 0.01 & 0.105 & 0.713 \\
Mesothelin & $13.9(0.03-99.97)$ & 1.66 & 3.93 & 17.1 \\
\hline
\end{tabular}

Numbers of CD3, CD8, CD20, and CD68+ cells per core, and percentage of totals cells positive for PD-L1, cells double positive for PD-L1 and CD68, cells positive for PD-L1 but negative for CD68, and cells positive for mesothelin as calculated by StrataQuest analysis software 
Table 3

Multivariate Analysis of Predictive Variables for Survival Outcomes

\begin{tabular}{|c|c|c|c|c|}
\hline \multicolumn{3}{|l|}{ Overall survival } & \multicolumn{2}{|l|}{$\underline{\text { Progression free survival }}$} \\
\hline Per unit HR (95 \% CI) & & $\mathbf{p}$ & Per unit HR (95 \% CI) & $\mathbf{p}$ \\
\hline KPS & $1.014(0.943,1.025)$ & 0.559 & Not included in model & - \\
\hline \multicolumn{5}{|l|}{ EOR } \\
\hline GTR & $1[\mathrm{ref}]$ & 0.207 & 1 [ref] & 0.439 \\
\hline STR & $1.905(0.708,5.747)$ & & $0.738(0.342,1.597)$ & \\
\hline \multicolumn{5}{|l|}{ Grade } \\
\hline 1 & 1 [ref] & 0.222 & $1[\mathrm{ref}]$ & $<0.001$ \\
\hline 2 & $0.654(0.165,4.421)$ & & $1.29 \times 10^{10}\left(5.684,4.45 \times 10^{64}\right)$ & \\
\hline 3 & $3.6 \times 10^{-10}(0,1.554)$ & & $4.77 \times 10^{10}\left(18.95,3.42 \times 10^{65}\right)$ & \\
\hline CD20+ & Not included in model & - & $0.966(0.887,0.999)$ & 0.041 \\
\hline PD-L1+/CD68- & $1.263(1.067,1.496)$ & 0.014 & Not included in model & - \\
\hline
\end{tabular}

$C I$ confidence interval, GTR gross total resection, $H R$ hazard ratio, ref reference, $S T R$ subtotal resection 